\title{
Anomalies in the One-Dimensional Anderson Model at Weak Disorder
}

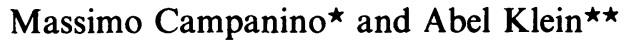 \\ Department of Mathematics, University of California, Irvine, \\ Irvine, CA 92717, USA
}

\begin{abstract}
We show that at the special energies $E=2 \cos \pi p / q$, the invariant measure, the Lyapunov exponent, and the density of states can be extended to zero disorder as $C^{\infty}$ functions in the disorder parameter. In particular, we obtain asymptotic series in the disorder parameter. This gives a rigorous proof of the existence of the anomalies originally discovered by Kappus and Wegner and studied by Derrida and Gardner and by Bovier and Klein.
\end{abstract}

\section{Introduction}

The one-dimensional Anderson model is given by the random Hamiltonian

where

$$
H=H_{0}+\lambda V \text { on } l^{2}(\mathrm{Z})
$$

$$
\left(H_{0} u\right)(n)=u(n+1)+u(n-1), \quad u \in \mathbf{Z},
$$

and the $V(x), x \in \mathbf{Z}$, are independent identically distributed random variables with common probability distribution $\mu$. We will write $h$ for the characteristic function of $\mu$, i.e. $h(t)=\int e^{-i t v} d \mu(v)$. The real parameter $\lambda$ will be called the disorder.

In this article we will always assume that $\mu$ has finite moments of all orders and is not concentrated in a single point; we normalize $\mu$ by

$$
\int v d \mu(v)=0, \quad \int v^{2} d \mu(v)=1 .
$$

The eigenvalue equation associated with $H$ is

$$
u(n+1)+u(n-1)=(E-\lambda V(n)) u(n) .
$$

Setting

$$
Z(n)=\frac{u(n)}{u(n-1)} \in \dot{\mathbf{R}}=\mathbf{R} \cup\{\infty\}
$$

* Permanent address: Dipartimento di Matematica, Universita de Bologna, p.zz.a S. Donato, 5, I-40126 Bologna, Italy

$\star \star$ Partially supported by NSF grant DMS 87-02301 
we may rewrite (1.1) as a recursion relation for $Z(n)$ :

$$
Z(n+1)=E-\lambda V(n)-\frac{1}{Z(n)} .
$$

This relation defines a Markov process. It is a theorem of Fürstenberg (e.g., [1]) that, if $\lambda \neq 0$, for each $E \in \mathbf{R}$ there exists a unique invariant probability measure $v_{\lambda, E}$ on $\mathbf{R}$, i.e. $v_{\lambda, E}$ satisfies

$$
\int_{\dot{\mathbf{R}}} f(x) d v_{\lambda, E}(x)=\int_{\mathbf{R}}\left[\int_{\dot{\mathbf{R}}} f\left(E-\lambda v-\frac{1}{x}\right) d v_{\lambda, E}(x)\right] d \mu(v)
$$

for all bounded measurable functions $f$. In addition, $v_{\lambda, E}$ is always a continuous measure and hence it can be viewed simply as a measure on $\mathbf{R}$. Notice that $v_{\lambda, E}$ is, up to multiplication by a constant, the only signed measure satisfying (1.2) (see [1]).

The Lyapunov exponent $\gamma_{\lambda}(E)$ and the integrated density of state $N_{\lambda}(E)$ can be obtained from the invariant measure by (e.g., [1])

$$
\begin{gathered}
\gamma_{\lambda}(E)=\int_{\mathbf{R}} \log |x| d v_{\lambda, E}(x), \\
N_{\lambda}(E)=\int_{0}^{\infty} d v_{\lambda, E}(x) .
\end{gathered}
$$

Kappus and Wegner [2] discovered that there were problems in the naive perturbation expansion in $\lambda$ for the Lyapunov exponent, as proposed by Thouless [3]. They found that the leading coefficient in $\lambda$ was wrong at the center of the band $(E=0)$, and that the differentiated density of states $\left(\rho_{\lambda}(E)=(d / d E) N_{\lambda}(E)\right)$ exhibited a discontinuity at that energy. They called this phenomenon an anomaly.

Derrida and Gardner looked at the invariant measure and found anomalies not only at $E=0$ but also at $E= \pm 1$, in the next to the leading coefficient in Thouless' expansion. They conjectured that such anomalies should occur at all energies of the form $E=2 \cos \pi p / q$.

Bovier and Klein [5] gave a very detailed analysis of these anomalies and proved Derrida and Gardner's conjecture, at the level of formal perturbation theory. They showed that, if $E=2 \cos \pi p / q$, the naive perturbation expansion for the invariant measure has a divergent $q^{\text {th }}$ term. They also derived a modified perturbation expansion with finite coefficients of all orders; those differ from the naive ones only at order $\geqq q-2$.

In this article we give a rigorous proof of the existence of these anomalies. We show that the modified weak disorder expansions of Bovier and Klein are asymptotic to the invariant measure, Lyapunov exponent, and density of states, in the sense of being the corresponding Taylor series at zero disorder.

\section{Statement of Results}

If the single site potential probability distribution $\mu$ is such that its characteristic function and its derivative go to zero at infinity, Klein and Speis [6] have shown that the invariant measure $v_{\lambda, E}$ is absolutely continuous. Let $\varphi_{\lambda, E}$ be its density. 
Then $\varphi_{\lambda, E}(x) \geqq 0, \int \varphi_{\lambda, E}(x) d x=1$, and it follows from (1.2) that it must be the unique normalized solution of the equation

$$
\varphi_{\lambda, E}(x)=\int \varphi_{\lambda, E}\left(\frac{1}{E-\lambda v-x)}\right) \frac{1}{(E-\lambda v-x)^{2}} d \mu(v) .
$$

Following $[4,5]$ we write $E=E_{0}+\lambda^{2} \varepsilon$ and rewrite (2.1) as

$$
D_{\lambda, \varepsilon} S_{E_{0}} \varphi_{\lambda, E}=\varphi_{\lambda, E}
$$

where

$$
\begin{aligned}
\left(S_{E_{0}} f\right)(x) & =f\left(\frac{1}{E_{0}-x}\right) \frac{1}{\left(E_{0}-x\right)^{2}}, \\
D_{\lambda, \varepsilon} & =\int \exp \left[\lambda v \frac{d}{d x}-\lambda^{2} \varepsilon \frac{d}{d x}\right] d \mu(v)
\end{aligned}
$$

are bounded operators on $L^{1}(\mathbf{R}, d x), S_{E_{0}}$ being an isometry. We will also write $D_{\lambda}(E)=D_{\lambda, E / \lambda^{2}}$.

The properties of $S_{E_{0}}$ are responsible for the appearance of anomalies $[4,5]$. If $-2<E_{0}<2$, we can write $E_{0}=2 \cos \pi \alpha$ and, by a change of variables, $S_{E_{0}}$ is given by the shift of $\pi \alpha$ on the circle of length $\pi$. If $\alpha$ is irrational the equation

$$
S_{E_{0}} \varphi=\varphi
$$

has a unique normalized solution. But if $\alpha$ is rational, say $\alpha=p / q, 0<p<q$ relatively prime integers, then $S_{E_{0}}^{q}=I$ and its spectrum consists of the $q^{\text {th }}$ roots of unity, each with infinite multiplicity. In this case we have problems if we try to perturb (2.2) around $\lambda=0$.

To overcome this difficulty, let us replace $[4,5](2.2)$ by

$$
A_{\lambda, E_{0}, \varepsilon} \varphi_{\lambda, E_{0}, \varepsilon}=0,
$$

where $E_{0}=2 \cos \pi p / q, 0<p<q$ relatively prime integers, $\varphi_{\lambda, E_{0}, \varepsilon} \equiv \varphi_{\lambda, E_{0}+\lambda^{2} \varepsilon}$, and

$$
A_{\lambda, E_{0}, \varepsilon}=\frac{\left(D_{\lambda, \varepsilon} S_{E_{0}}\right)^{q}-I}{\lambda^{2}}
$$

with $\lambda \neq 0$.

Let $\mathscr{K}=L^{2}\left(\mathbf{R},\left(1+x^{2}\right) d x\right)$. Then $\mathscr{K}$ is continuously imbedded in $L^{1}(\mathbf{R}, d x)$ and $D_{\lambda, \varepsilon}$ and $S_{E_{0}}$ are bounded operators on $\mathscr{K}$ with $S_{0}$ unitary on $\mathscr{K}$. It will be convenient to consider (2.2) and (2.4) on $\mathscr{K}$. Under the assumptions outlined above $\varphi_{\lambda, E} \in \mathscr{K}$ for all $\lambda \neq 0$ and $E \in \mathbf{R}[6]$.

Bovier and Klein showed that we can take the limit as $\lambda \rightarrow 0$ in (2.5) obtaining the differential operator

$$
A_{0, E_{0}, \varepsilon}=\sum_{k=0}^{q-1} S_{E_{0}}^{k}\left(\frac{1}{2} \frac{d^{2}}{d x^{2}}-\varepsilon \frac{d}{d x}\right) S_{E_{0}}^{q-k} .
$$

They showed that $A_{\lambda, E_{0}, \varepsilon} \rightarrow A_{0, E_{0}, \varepsilon}$ strongly in $\mathscr{K}$ on a core for $A_{0, E_{0}, \varepsilon}$, and that $A_{0, E_{0}, \varepsilon}$ has zero as an isolated simple eigenvalue. In fact they solved the equation

$$
A_{0, E_{0}, \varepsilon} \varphi_{0, E_{0}, \varepsilon}=0
$$


In $\mathscr{K}$ obtaining the unique (normalized by $\int \varphi_{0, E_{0, \varepsilon}}(x) d x=1$ ) solution

$$
\varphi_{0,0, \varepsilon}=N_{0, \varepsilon}\left(1+x^{4}\right)^{-1 / 2} \exp \left(\sqrt{2} \varepsilon \arctan \left[\sqrt{2} x\left(1-x^{2}\right)^{-1}\right]\right),
$$

if $E_{0}=0$, and

$$
\begin{aligned}
\varphi_{0, E_{0}, \varepsilon}= & N_{E_{0}, \varepsilon}\left(x^{2}-E_{0} x+1\right)^{-1} \exp \left(K_{E_{0}} \varepsilon\left(4-E_{0}^{2}\right)^{-1 / 2}\right. \\
& \cdot \arctan \left[\left(2 x-E_{0}\right)\left(4-E_{0}^{2}\right)^{-1 / 2}\right]
\end{aligned}
$$

if $E_{0} \neq 0$. Here $N_{E_{0}, \varepsilon}$ is a normalization constant and $K_{E_{0}, \varepsilon}$ is some constant that can be computed. They defined Hilbert spaces

$$
\mathscr{K}_{E_{0, \varepsilon}}=L^{2}\left(\mathbf{R}, \varphi_{0, E_{0}, \varepsilon}(x)^{-1} d x\right),
$$

which are just $\mathscr{K}$ equipped with an equivalent norm, and showed that $A_{0, E_{0, \varepsilon}}$ is a self-adjoint operator on $\mathscr{K}_{E_{0, \varepsilon},}$ with discrete spectrum, $A_{0, E_{0}, \varepsilon} \leqq 0$, with 0 an isolated simple eigenvalue with eigenvector $\varphi_{0, E_{0}, \varepsilon}$.

In addition, Bovier and Klein derived a uniquely defined formal perturbation series in $\lambda$ for $\varphi_{\lambda, E_{0}, \varepsilon}$ in $\mathscr{K}$. More precisely, if we formally write

$$
\varphi_{\lambda, E_{0}, \varepsilon}=\sum_{n=0}^{\infty} \frac{\lambda^{n}}{n !} \varphi_{0, E_{0}, \varepsilon}^{(n)}
$$

it follows from (2.5) that

$$
\left(\begin{array}{l}
n \\
2
\end{array}\right) A_{0, E_{0, \varepsilon}} \varphi_{0, E_{0}, \varepsilon}^{(n-2)}=-\sum_{k=3}^{n}\left(\begin{array}{l}
n \\
k
\end{array}\right)\left[\frac{d^{k}}{d \lambda^{k}}\left(D_{\lambda, \varepsilon} S_{E_{0}}\right)^{q}\right]_{\lambda=0} \varphi_{0, E_{0}, \varepsilon}^{(n-k)} .
$$

Bovier and Klein [5] proved that these equations have a unique normalized set of solutions $\varphi_{0, E_{0}, \varepsilon}^{(n)}$ in the domain of $A_{0, E_{0}, \varepsilon}$ in $\mathscr{K}_{E_{0, \varepsilon}}$ and hence in $\mathscr{K}$.

Our main result is

Theorem 2.1. Let the single site probability distribution $\mu$ have finite moments of all orders, with zero mean and variance one. Suppose its characteristic function $h$ is $O\left(\left[\left(1+t^{2}\right)^{-\alpha / 2}\right]\right.$ as $t \rightarrow \infty$ for some $\alpha>0$. Let $E_{0}=2 \cos \pi p / q$, with $0<p<q$ relatively prime integers, and let $\varepsilon \in \mathbf{R}$. Then the map $\mathbf{R} \ni \lambda \rightarrow \varphi_{\lambda, E_{0}, \varepsilon} \in \mathscr{K}$ is $C^{\infty}$. Moreover, its Taylor series at $\lambda=0$ is given by (2.8) and (2.9).

It follows from [7,6] that the map is $C^{\infty}$ on $\mathbf{R} \backslash\{0\}$. The point of the Theorem is that the map is $C^{\infty}$ at $\lambda=0$ after we extend $\varphi_{\lambda, E_{0}, \varepsilon}$ to $\lambda=0$ by (2.6) and (2.7).

It is easy to see that (1.3) and (1.4) express $\gamma_{\lambda}(E)$ and $N_{\lambda}(E)$ as values of continuous linear functionals on $\mathscr{K}$ applied to $\varphi_{\lambda, E}$. Thus we have

Corollary 2.2. Under the above hypotheses the maps $\lambda \rightarrow \gamma_{\lambda}\left(E_{0}\right)$ and $\lambda \rightarrow N_{\lambda}\left(E_{0}\right)$ extend to $\lambda=0$ as $C^{\infty}$ functions.

Bovier and Klein [5] showed that the spectrum of $A_{\lambda, \varepsilon}$ in $\mathscr{K}$ is contained in the set $\left\{z ; \operatorname{Re} z \leqq-a_{1}\right\} \cup\{0\}$ for some $a_{1}>0$ and all $\lambda \neq 0$ sufficiently small. They also proved that $\left(A_{\lambda, \varepsilon}-z\right)^{-1} \rightarrow\left(A_{0, \varepsilon}-z\right)^{-1}$ strongly in $\mathscr{K}$ for $\operatorname{Re} z>a_{2}$ for some $a_{2}>0$. But the proof of Theorem 2.1 requires more. To use perturbation theory we need to show that $\left(A_{\lambda, \varepsilon}-z\right)^{-1} \rightarrow\left(A_{0, \varepsilon}-z\right)^{-1}$ strongly in $\mathscr{K}$ for all $z$ with $|z|=a_{3}$, for some $0<a_{3}<a_{1}$. 
In the next section we will describe the proof of Theorem 2.1 in the center of the band $\left(E_{0}=0\right)$. We start by rewriting (2.2) in the language of [7], so we can use the technical machinery we had developed. The key lemma is Lemma 3.2, (3.3) being the crucial estimate. Lemma 3.3 is a simple consequence of Lemma 3.2. The estimates in Lemmas 3.2 and 3.3 enable us to prove Lemma 3.4.

In Sect. 4 we outline the modifications required for general $E_{0}=2 \cos \pi p / q$.

\section{The Center of the Band}

In [7] we introduced the Hilbert spaces

$$
\begin{aligned}
\mathscr{H}_{0}= & \left\{f:[0, \infty) \rightarrow \mathbf{C} \text { measurable; }\|f\|_{0}=\left\|r^{-1 / 2} f\left(r^{2}\right)\right\|_{2}<\infty\right\}, \\
\mathscr{H}_{n}= & \{f:[0, \infty) \rightarrow \mathbf{C} \text { continuous, } f(n-1) \text {-times differentiable on }(0, \infty) \text { with } \\
& f^{(n-1)} \text { absolutely continuous with }
\end{aligned}
$$

$$
\left.\|f\|_{\mathscr{H}_{n}}^{2}=\sum_{m=1}^{n} \sum_{k=0}^{m}\left\|2^{k} r^{m-1 / 2} f^{(k)}\left(r^{2}\right)\right\|_{2}^{2}<\infty\right\}
$$

for $n=1,2, \ldots$, and

$$
\mathscr{H}_{0}^{0}=\mathscr{H}_{0}, \quad \mathscr{H}_{n}^{0}=\left\{f \in \mathscr{H}_{n} ; f(0)=0\right\}, \quad n=1,2, \ldots,
$$

the $L^{2}$ norms being in $L^{2}([0, \infty), d r)$.

We also introduced operators $T$ and $R$ given by

$$
\begin{aligned}
& (T f)\left(r^{2}\right)=-2 \int_{0}^{\infty} J_{0}(r s) f^{\prime}\left(s^{2}\right) s d s, \\
& (R f)\left(r^{2}\right)=r \int_{0}^{\infty} J_{-1}(r s) f\left(s^{2}\right) d s
\end{aligned}
$$

where $J_{n}$ is the Bessel function of order $n$. We showed that $T$ is unitary on $\mathscr{H}_{n}$ for $n=1,2, \ldots, R$ is unitary on $\mathscr{H}_{n}^{0}$ for $n=0,1, \ldots$, and $T=R$ on $\mathscr{H}_{n}^{0}$, so $T$ leaves $\mathscr{H}_{n}^{0}$ invariant.

Let $\beta_{\lambda}(r ; E)=h((\lambda / 2) r) e^{(i / 2) E r}$. Then $B_{\lambda}(E)$ will denote the operator multiplication by $\beta_{\lambda}(\cdot E)$, i.e.,

$$
\left(B_{\lambda}(E) f\right)\left(r^{2}\right)=\beta_{\lambda}\left(r^{2} ; E\right) f\left(r^{2}\right) .
$$

Notice that, under the hypotheses of Theorem 2.1 (see [7; Lemma 7.1]), $B_{\lambda}(E)$ is a bounded operator in all $\mathscr{H}_{n}, n=0,1, \ldots$, leaving $\mathscr{H}_{n}^{0}$ invariant.

In [7] we studied the eigenvalue equation

$$
B_{\lambda}(E) T \xi_{\lambda, E}=\xi_{\lambda, E} \text {. }
$$

Under the hypotheses of Theorem 2.1 we proved that, if $\lambda \neq 0,(3.1)$ has a unique solution $\xi_{\lambda, E} \in \mathscr{H}_{n}$ normalized by $\xi_{\lambda, E}(0)=1$, and that 1 is an isolated simple eigenvalue for $B_{\lambda}(E) T$ in each $\mathscr{H}_{n}$. We also proved that $B_{\lambda}(E) R B_{\lambda}(E)$ has spectral radius less than one in each $\mathscr{H}_{n}^{0}$ by an explicit estimate. It is also proved in $[8,6]$ that $B_{\lambda}(E) T B_{\lambda}(E)$ is a compact operator in each $\mathscr{H}_{n}$.

The eigenvector $\xi_{\lambda, E}$ is related to the invariant measure $v_{\lambda, E}$, and Eq. (3.1) is 
related to Eq. (2.2). Klein and Speis [6] showed that $\xi_{\lambda, E}$ is the Fourier transform of the density $\varphi_{\lambda, E}$ of $v_{\lambda, E}$, i.e.,

$$
\xi_{\lambda, E}\left(r^{2}\right)=\int e^{(i / 2) r^{2} x} \varphi_{\lambda, E}(x) d x .
$$

More precisely, let

$$
(\mathscr{F} g)\left(r^{2}\right)=\frac{1}{4 \sqrt{\pi}} \int e^{(i / 2) r^{2} x} g(x) d x,
$$

and consider the real Hilbert spaces

$$
\begin{aligned}
\mathscr{K}^{\prime} & =\{g \in \mathscr{K} ; g \text { real valued }\}, \\
\mathscr{H}^{\prime} & =\left\{f \in \mathscr{H}_{1} ; f(0) \in \mathbf{R}\right\},
\end{aligned}
$$

where $\mathscr{H}_{1}^{\prime}$ is equipped with the inner product $\left\langle f_{1}, f_{2}\right\rangle_{\mathscr{H}_{1}^{\prime}}=\operatorname{Re}\left\langle f_{1}, f_{2}\right\rangle_{\mathscr{H}_{1}}$. Then $\mathscr{F}$ is an orthogonal map from $\mathscr{K}^{\prime}$ to $\mathscr{H}_{1}^{\prime}$. Moreover [6], $\mathscr{K}^{\prime}$ and $\mathscr{H}_{1}^{\prime}$ are invariant under $S_{0}, D_{\lambda}(E)$, and $T, B_{\lambda}(E)$, respectively, and

$$
\begin{aligned}
T & =\mathscr{F} S_{0} \mathscr{F}^{-1}, \\
B_{\lambda}(E) & =\mathscr{F} D_{\lambda}(E) \mathscr{F}^{-1},
\end{aligned}
$$

and hence (3.1) is just (2.2), with

$$
\xi_{\lambda, E}=4 \sqrt{\pi} \mathscr{F} \varphi_{\lambda, E} .
$$

We will abuse the notation by writing $A_{\lambda, \varepsilon}$ for $\mathscr{F} A_{\lambda, 0, \varepsilon} \mathscr{F}^{-1}$. Then we have

$$
A_{\lambda, \varepsilon}=\frac{\left(B_{\lambda, \varepsilon} T\right)^{2}-I}{\lambda^{2}}, \quad A_{\lambda, \varepsilon} \xi_{\lambda, \varepsilon}=0
$$

0 being an isolated simple eigenvalue, where $B_{\lambda, \varepsilon}=B_{\lambda}\left(\lambda^{2} \varepsilon\right), \xi_{\lambda, \varepsilon}=\xi_{\lambda, \lambda^{2} \varepsilon}$.

We will now study the behavior of $B_{\lambda, \varepsilon} T$ for small $\lambda$. We assume the hypothesis of Theorem 2.1 .

Lemma 3.1. There exists $c_{1}>0$ and $\lambda_{0}>0$ such that

$$
\left\|\left(B_{\lambda, \varepsilon} T\right)^{2}\right\|_{\mathscr{H}_{0} \rightarrow \mathscr{H}_{0}} \leqq e^{-c_{1} \lambda^{2}}
$$

for all $\varepsilon>0$ and $\lambda$ with $|\lambda|<\lambda_{0}$.

Proof. By Lemma 3.1 of [7] we have

$$
\left\|\left(B_{\lambda, \varepsilon} T\right)^{2}\right\|_{\mathscr{H}_{0} \rightarrow \mathscr{H}_{0}} \leqq\left\|h\left(\frac{\lambda}{2} r^{2}\right)\right\|_{p}^{2}
$$

for any $p$ with $2<p<\infty$, where the $L^{p}$ space is $L^{p}([0, \infty), d r)$. If we make the choice $p=c_{2} / \lambda^{2}$ for a sufficiently large constant $c_{2}$ we get (3.2).

Lemma 3.2. Let $\varepsilon>0$ be fixed. Then for any $n=1,2, \ldots$ there exists a constant $M_{n}$ such that

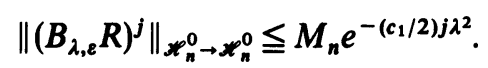

for any $\lambda$ with $|\lambda| \leqq \lambda_{1}$ for some $\lambda_{1}>0$. 
Proof. By an explicit estimate it is easy to see that for any $\varepsilon>0$ and any $n=1,2, \ldots$, there exists $c_{3, n}=c_{3, n}(\varepsilon)<\infty$ such that

$$
\left\|B_{\lambda, \varepsilon}\right\|_{\mathscr{H}_{n} \rightarrow \mathscr{X}_{n}} \leqq e^{c_{3, n} \lambda^{2}} \text {. }
$$

Since $T$ is unitary on all $\mathscr{H}_{n}$ the bound of (3.4) also holds for $B_{\lambda, \varepsilon} T$.

To prove the lemma we apply an interpolation argument similar to the one used in the proof of Theorem 5.1 in [7]. We introduce a family of operators $S(z)$ depending on a complex variable $z$, defined on the strip $0 \leqq \operatorname{Re} z \leqq 1$. $S(z)$ is the operator given by multiplication by the function

$$
e^{z^{2}} h\left(\frac{\lambda}{2} r^{2}\right) e^{(i / 2) \varepsilon \lambda^{2} r^{2}}\left(1+r^{4}\right)^{\alpha\left(\dot{\lambda}^{2}-z\right)},
$$

where $\alpha<\gamma / 4$. Here $\gamma>0$ is such that $|h(t)| \leqq\left(1+t^{2}\right)^{-\gamma}$ for all $t \geqq 0$. Notice that such a $\gamma$ always exists by our hypotheses.

We define $K(z)=(S(z) R)^{k}$, where $k$ is such that $K(1+i y)$ is bounded from $\mathscr{H}_{0}$ to $\mathscr{H}_{n+1}^{0}$ for all $\lambda$ sufficiently small with a norm uniformly bounded in $\lambda$. The existence of such a $k$ follows from Theorem 5.1 in [7]).

On the other hand we have

$$
\|S(i y)\|_{\mathscr{H}_{0} \rightarrow \mathscr{X}_{0}} \leqq e^{-y^{2}} \sup _{r \geqq 0}\left|h\left(\frac{\lambda}{2} r^{2}\right)\left(1+r^{4}\right)^{\alpha \lambda^{2}}\right| \leqq 1
$$

for $\lambda$ sufficiently small, since $|h(t)| \leqq\left(1+t^{2}\right)^{-\gamma},\left(1+t^{2}\right)^{\sigma} \leqq\left(1+\sigma t^{2}\right)$ for $0<\sigma \leqq 1$, and $\alpha<\gamma / 4$. Thus it follows that

$$
\|K(i y)\|_{\mathscr{H}_{0} \rightarrow \mathscr{H}_{0}}=\left\|(S(i y) R)^{k}\right\|_{\mathscr{H}_{0} \rightarrow \mathscr{H}_{0}} \leqq 1
$$

since $R$ is a unitary operator on $\mathscr{H}_{0}$.

We also have that

$$
\sup _{0 \leqq \operatorname{Re} z \leqq 1}\|K(z)\|_{\mathscr{H}_{0} \rightarrow \mathscr{H}_{0}}<\infty
$$

and $K(z)$ depends analytically on $z$ for $0<\operatorname{Re} z<1$ and is continuous in $z$ for $0 \leqq \operatorname{Re} z \leqq 1$ as a family of bounded operators from $\mathscr{H}_{0}$ to $\mathscr{H}_{0}$. We can then apply the Calderon-Lions interpolation theorem (e.g., [9]) to the family $K(z)$ getting

$$
\left\|K\left(\lambda^{2}\right)\right\|_{\mathscr{H}_{0} \rightarrow\left[\mathscr{H}_{0}, \mathscr{H}_{n+1}^{0}\right] \lambda^{2}} \leqq e^{c_{4} \lambda^{2}},
$$

where $c_{4}$ is some constant independent of $\lambda$, for all $\lambda$ sufficiently small. Here, we used the notation that when we are interpolating in $t$ between the spaces $X_{0}$ and $X_{1}$ we denote the interpolating spaces by $\left[X_{0}, X_{1}\right]_{t}$. We will also write $\left[X_{0}, X_{1}\right]_{t}^{m+1}$ for $\left[\left[X_{0}, X_{1}\right]_{t}^{m}, X_{1}\right]_{t}$.

We now apply again an interpolation argument to the fixed operator $K\left(\lambda^{2}\right)=$ $\left(e^{\lambda^{2}} B_{\lambda, \varepsilon} R\right)^{k}$. We observe that by (3.4)

$$
\left\|K\left(\lambda^{2}\right)\right\|_{\mathscr{H}_{n+1}^{0} \rightarrow \mathscr{X}_{n+1}^{0}} \leqq e^{c_{5} \lambda^{2}}
$$

with $c_{5}=k\left(c_{3, n+1}+1\right)$ so that by interpolating between (3.5) and (3.6) we get

$$
\left\|K\left(\lambda^{2}\right)\right\|_{\left[\mathscr{H}_{0}, \mathscr{H}_{n+1}^{0}\right]_{\lambda^{2}} \rightarrow\left[\mathscr{H}_{0}, \mathscr{H}_{n+1}^{0}\right]_{\lambda^{2}}^{2} \leqq e^{c_{6} \lambda^{2}}}
$$


with $c_{6}$ the largest of $c_{4}$ and $c_{5}$. Iterating this procedure we get

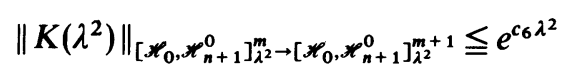

for $m=1,2, \ldots$, so that

$$
\left\|K\left(\lambda^{2}\right)^{j}\right\|_{\mathscr{H}_{0} \rightarrow\left[\mathscr{H}_{0}, \mathscr{x}_{n+1}^{0}\right]_{\lambda^{2}}^{j}} \leqq e^{c_{6} j \lambda^{2}} .
$$

But, by Lemma 5.1 (see Appendix A), there exists $0<c<\infty$ such that

$$
\left[\mathscr{H}_{0}, \mathscr{H}_{n+1}^{0}\right]_{\lambda^{2}}^{\left[c(n+1) / \lambda^{2}\right]}
$$

is continuously imbedded into $\mathscr{H}_{n}^{0}$, the injection being a contraction, so that

$$
\left\|K\left(\lambda^{2}\right)^{\left[c(n+1) / \lambda^{2}\right]}\right\|_{\mathscr{H}_{0} \rightarrow \mathscr{H}_{n}^{0}} \leqq e^{c_{6}\left[c(n+1) / \lambda^{2}\right] \lambda^{2}} \leqq e^{c_{7}} .
$$

We can now prove (3.3). Indeed, if $j<\left[c(n+1) / \lambda^{2}\right] k+1$, then by (3.4) we have

$$
\left\|\left(B_{\lambda, \varepsilon} R\right)^{j}\right\|_{\mathscr{H}_{n}^{0} \rightarrow \mathscr{X}_{n}^{0}} \leqq e^{c_{3, n} j \lambda^{2}} .
$$

If $j \geqq\left[c(n+1) / \lambda^{2}\right] k+1$ we can write

$$
\begin{aligned}
&\left\|\left(B_{\lambda, \varepsilon} R\right)^{j}\right\|_{\mathscr{H}_{n}^{0} \rightarrow \mathscr{H}_{n}^{0}} \leqq\left\|\left(B_{\lambda, \varepsilon} R\right)^{j}\right\|_{\mathscr{H}_{0} \rightarrow \mathscr{H}_{n}^{0}} \\
& \leqq\left\|\left(B_{\lambda, \varepsilon} R\right)^{j-\left[c(n+1) / \lambda^{2}\right] k}\right\|_{\mathscr{H}_{0} \rightarrow \mathscr{H}_{0}}\left\|\left(B_{\lambda, \varepsilon} R\right)^{\left[c(n+1) / \lambda^{2}\right] k}\right\|_{\mathscr{H}_{0} \rightarrow \mathscr{H}_{n}^{0}} \\
& \leqq e^{-\left(c_{1} / 2\right) \lambda^{2}\left(j-1-\left[c(n+1) / \lambda^{2}\right] k\right)+c_{7}} \\
&=e^{-\left(c_{1} / 2\right) j \lambda^{2}} e^{\left(c_{1} / 2\right) \lambda^{2}\left(1+\left[c(n+1) / \lambda^{2}\right] k\right)+c_{7}} \\
& \leqq \bar{M} e^{-\left(c_{1} / 2\right) \lambda^{2} j}
\end{aligned}
$$

for some constant $\bar{M}$ independent of $\lambda$. Notice we used (3.2) and (3.7).

Putting together (3.8) and (3.9) we get (3.3) with

$$
M_{n}=\max \left(\bar{M}, e^{c(n+1)\left(c_{3, n}+c_{1} / 2\right)}\right) \text {. }
$$

Lemma 3.3. For any $n=1,2, \ldots$, for all $\lambda \neq 0$ sufficiently small and all $\varepsilon$ we have: (i) the spectrum of $A_{\lambda, \varepsilon}$ restricted to $\mathscr{H}_{n}^{0}$ is contained in $\left\{z ; \operatorname{Re} z \leqq-c_{1} / 2\right\}$.

(ii)

$$
\left\|e^{t A_{\lambda, \varepsilon}}\right\|_{\mathscr{H}_{n}^{0} \rightarrow \mathscr{H}_{n}^{0}} \leqq M_{n} e^{-\left(c_{1} / 2\right) t}
$$

for all $t \geqq 0$.

(iii)

$$
\left\|\left(A_{\lambda, \varepsilon}-z\right)^{-1}\right\|_{\mathscr{H}_{n}^{0} \rightarrow \mathscr{H}_{n}^{0}} \leqq M_{n}\left(\operatorname{Re} z+\frac{c_{1}}{2}\right)^{-1}
$$

if $\operatorname{Re} z>-c_{1} / 2$.

Proof. (i) follows form Lemma 3.2. To prove (ii), notice that we have, using Lemma 3.2, that

$$
\begin{aligned}
\left\|e^{t A_{\lambda, \varepsilon}}\right\|_{\mathscr{H}_{n}^{0} \rightarrow \mathscr{H}_{n}^{0}} & =e^{-t / \lambda^{2}}\left\|e^{\left(t / \lambda^{2}\right)\left(B_{\lambda, e} R\right)^{2}}\right\|_{\mathscr{H}_{n}^{0} \rightarrow \mathscr{H}_{n}^{0}} \\
& \leqq \sum_{j=0}^{\infty} \frac{1}{j !}\left(\frac{t}{\lambda^{2}}\right)^{j} e^{-t / \lambda^{2}}\left\|\left(B_{\lambda, \varepsilon} R\right)^{2 j}\right\|_{\mathscr{H}_{n}^{0} \rightarrow \mathscr{X}_{n}^{0}}
\end{aligned}
$$




$$
\begin{aligned}
& \leqq M_{n} \sum_{j=0}^{\infty} \frac{1}{j !}\left(\frac{t}{\lambda^{2}}\right)^{j} e^{-t / \lambda^{2}-j c_{1} \lambda^{2}} \\
& =M_{n} e^{\left(t / \lambda^{2}\right)\left(e^{-c_{1} \lambda^{2}}-1\right)} \\
& \leqq M_{n} e^{-\left(c_{1} / 2\right) t}
\end{aligned}
$$

for all $\lambda$ sufficiently small.

Now, if $\operatorname{Re} z>-c_{1} / 2$, we can compute the resolvent by

$$
\left(A_{\lambda, \varepsilon}-z\right)^{-1}=-\int_{0}^{\infty} e^{t A_{\lambda, \varepsilon}} e^{-z t} d t
$$

so using (3.11) we get (3.12).

Lemma 3.4. $\left(A_{\lambda, \varepsilon}-z\right)^{-1} \rightarrow\left(A_{0, \varepsilon}-z\right)^{-1}$ strongly in $\mathscr{H}_{n}, n=1,2, \ldots$, as $\lambda \rightarrow 0$, for any $z$ such that $z \neq 0$ and $\operatorname{Re} z>-c_{1} / 2$.

Proof. Bovier and Klein [5] proved that $\left(A_{\lambda, \varepsilon}-z\right)^{-1} \rightarrow\left(A_{0, \varepsilon}-z\right)^{-1}$ strongly in $\mathscr{H}_{1}$ for $\operatorname{Re} z>2 c_{3,1}$, where $c_{3,1}$ is given in (3.4). We give their argument for completeness. Let $f \in \mathscr{H}_{1}$, we have

$$
\left(A_{\lambda, \varepsilon}-z\right) f=\lambda^{-2}\left[\left(1+\lambda^{2} z\right)-\left(B_{\lambda, \varepsilon} T^{2}\right] f,\right.
$$

so for $\operatorname{Re} z>0$ we get, using (3.4), that $\left\|\left(A_{\lambda, \varepsilon}-z\right) f\right\|_{\mathscr{H}_{1}} \geqq \lambda^{-2}\left[1+\lambda^{2} \operatorname{Re} z-e^{c \lambda^{2}}\right]\|f\|_{\mathscr{H}_{1}}$ with $c=2 c_{3,1}$. Thus, if $\operatorname{Re} z>c$ we get

$$
\left\|\left(A_{\lambda, \varepsilon}-z\right)^{-1}\right\|_{\mathscr{H}_{1} \rightarrow \mathscr{H}_{1}} \leqq 2(\operatorname{Re} z-c)^{-1}
$$

for all $\lambda$ sufficiently small. The desired convergence now follows from the resolvent equation since $A_{\lambda, \varepsilon} \rightarrow A_{0, \varepsilon}$ strongly in $\mathscr{H}_{1}$ on a core for $A_{0, \varepsilon}$.

Since $A_{0, \varepsilon}$ is a negative self-adjoint operator on $\mathscr{H}_{1}$ equipped with an equivalent norm [5], it generates a strongly continuous semigroup $e^{t A_{0, \varepsilon}}$ in $\mathscr{H}_{1}$ with $\left\|e^{t A_{0, \varepsilon}}\right\|_{\mathscr{H}_{1} \rightarrow \mathscr{H}_{1}}<C$ for all $t \geqq 0$ for some constant $C<\infty$. We can then apply the Trotter-Kato-Neveu Theorem (see [10]) to get that $e^{t A_{\lambda, \varepsilon}} \rightarrow e^{t A_{0, \varepsilon}}$ strongly in $\mathscr{H}_{1}$ as $\lambda \rightarrow 0$ for all $t \geqq 0$, uniformly for $t$ in a compact interval. Moreover, by Theorem 1.1 in [10] we see that

$$
\left(B_{\sqrt{t / k, \varepsilon}} T\right)^{2 k} \rightarrow e^{t A_{0, \varepsilon}}
$$

strongly in $\mathscr{H}_{1}$ as $k \rightarrow \infty$, uniformly for $t$ in compact intervals.

Actually, $e^{t A_{0, \varepsilon}}$ is a strongly continuous uniformly bounded semigroup on each $\mathscr{H}_{n}, n=1,2, \ldots$ Indeed, let $\varphi \in \mathscr{H}_{n+2}$, then the set $\left\{\left(B_{\sqrt{t / k, \varepsilon}} T\right)^{2 k} \varphi ; k=1,2, \ldots\right\}$ is uniformly bounded in $\mathscr{H}_{n+2}$ by (3.4). But bounded sets of $\mathscr{H}_{n+2}$ are precompact in $\mathscr{H}_{n}$ (see Appendix B). Since we have convergence as $k \rightarrow \infty$ in $\mathscr{H}_{1}$, and convergence in $\mathscr{H}_{n+2}$ implies convergence in $\mathscr{H}_{1}$ we can conclude that $\left(B_{\sqrt{t / k, \varepsilon}} T\right)^{2 k} \varphi \rightarrow e^{t A_{0, \varepsilon}} \varphi$ in $\mathscr{H}_{n}$ for each $\varphi \in \mathscr{H}_{n+2}$. The uniform bound gives the strong convergence in $\mathscr{H}_{n}$. Similarly one proves the strong continuity of $e^{t A_{0, \varepsilon}}$ in $\mathscr{H}_{n}$, and that

$$
e^{t A_{\lambda, \varepsilon}} \rightarrow e^{t A_{0, \varepsilon}} \text { strongly in } \mathscr{H}_{n} .
$$

As before, let $\xi_{\lambda, \varepsilon}$ denote the unique solution of $A_{\lambda, \varepsilon} \xi_{\lambda, \varepsilon}=0$, normalized by 
$\xi_{\lambda, \varepsilon}(0)=1$. Let $P_{\lambda, \varepsilon}$ be the corresponding spectral projection (recall 0 is an algebraically simple eigenvalue of $A_{\lambda, \varepsilon}$, all $\lambda \in \mathbf{R}$ and $\varepsilon$ ).

By (3.13),

$$
\left(B_{\sqrt{t / k, \varepsilon}} T\right)^{2 m k} \xi_{0, \varepsilon} \rightarrow e^{m t A_{0, \varepsilon}} \xi_{0, \varepsilon} \quad \text { as } \quad k \rightarrow \infty
$$

We can write

$$
\left(B_{\sqrt{t / k, \varepsilon}} T\right)^{2 m k} \xi_{0, \varepsilon}=\xi_{\sqrt{t / k, \varepsilon}}+\left(B_{\sqrt{t / k, \varepsilon}} T\right)^{2 m k}\left(\xi_{0, \varepsilon}-\xi_{\sqrt{t / k}, \varepsilon}\right)
$$

so

$$
\left[\xi_{0, \varepsilon}-\xi_{\sqrt{t / k, \varepsilon}}\right]-\left(B_{\sqrt{t / k, \varepsilon}} T\right)^{2 m k}\left(\xi_{0, \varepsilon}-\xi_{\sqrt{t / k, \varepsilon}}\right) \rightarrow 0
$$

in $\mathscr{H}_{n}$ as $k \rightarrow \infty$.

But $\xi_{0, \varepsilon}-\xi_{\sqrt{t / k, \varepsilon}} \in \mathscr{H}_{n}^{0}$, and by Lemma 3.2 we can choose $m$ such that

$$
\left\|\left(B_{\sqrt{t / k, \varepsilon}} T\right)^{2 m k}\right\|_{\mathscr{H}_{n}^{0} \rightarrow \mathscr{X}_{n}^{0}}<\frac{1}{2}
$$

for all $k$ sufficiently large, so (3.14) implies that $\xi_{\sqrt{t / k, \varepsilon}} \rightarrow \xi_{0, \varepsilon}$ in $\mathscr{H}_{n}$ as $k \rightarrow \infty$. Since the convergence in (3.13) is uniform for $t$ in compact intervals, and so is the bound obtained from (3.3), it follows that $\xi_{\lambda, \varepsilon} \rightarrow \xi_{0, \varepsilon}$ in $\mathscr{H}_{n}$ as $\lambda \rightarrow 0$. This implies that $P_{\lambda, \varepsilon} \rightarrow P_{0, \varepsilon}$ in norm as operators in $\mathscr{H}_{n}$, since $P_{\lambda, \varepsilon} f=f(0) \xi_{\lambda, \varepsilon}$.

It follows from Lemma 3.3 and (3.13) that if $\operatorname{Re} z>-c_{1} / 2$ and $z \neq 0$, then $z$ is in the resolvent of $A_{0, \varepsilon}$ as an operator in $\mathscr{H}_{n}$. Indeed, we can write

$$
\left(A_{0, \varepsilon}-z\right)^{-1}=-z^{-1} P_{0, \varepsilon}+\int_{0}^{\infty} e^{t A_{0, \varepsilon}} e^{-z t}\left(I-P_{0, \varepsilon}\right) d t,
$$

where the integral converges in norm by (3.11), (3.13) and (3.14), and the fact that $I-P_{0, \varepsilon}$ projects onto $\mathscr{H}_{n}^{0}$.

Thus, if $\operatorname{Re} z>-c_{1} / 2, z \neq 0$, and $f \in \mathscr{H}_{n}$, we have

$$
\begin{aligned}
\left(A_{\lambda, \varepsilon}-z\right)^{-1} f-\left(A_{0, \varepsilon}-z\right)^{-1} f \\
=\frac{1}{z}\left(P_{0, \varepsilon}-P_{\lambda, \varepsilon}\right) f+\int_{0}^{T} e^{t A_{\lambda, \varepsilon}} e^{-z t}\left(P_{\lambda, \varepsilon}-P_{0, \varepsilon}\right) f d t \\
=\int_{0}^{T}\left(e^{t A_{\lambda, \varepsilon}}-e^{\left.t A_{0, \varepsilon}\right)}\right) P_{0, \varepsilon} f d t+\int_{T}^{\infty} e^{t A_{\lambda, \varepsilon}} e^{-z t}\left(I-P_{\lambda, \varepsilon}\right) f d t \\
\quad+\int_{T}^{\infty} e^{t A_{0, \varepsilon}} e^{-z t}\left(I-P_{0, \varepsilon}\right) f d t
\end{aligned}
$$

for any $T>0$. Given $\eta>0$, we first choose $\lambda_{1}>0$ such that if $|\lambda|<\lambda_{1}$, the first and the second term on the right-hand side of (3.16) have norm less that $\eta / 5$ (this can be done by the norm convergence of the projections plus (3.11)), uniformly in $T>0$. Then we choose $T$ so that the fourth and fifth terms have norm less than $\eta / 5$ (use (3.11), notice we have shown it also holds for $\lambda=0$ ). Finally we choose $0<\lambda_{2}<\lambda_{1}$ so that for $|\lambda|<\lambda_{2}$ the third term has norm less than $\eta / 5$ (use the strong convergence of the semigroups, which is uniform on compact intervals). This proves Lemma 3.4. 
We are now ready to prove Theorem 2.1 for $E_{0}=0$. Let $\Gamma$ be the circle $|z|=c_{1} / 4$. It follows from Lemma 3.3 that

$$
P_{\lambda, \varepsilon}=\frac{1}{2 \pi i} \int_{\Gamma}\left(A_{\lambda, \varepsilon}-z\right)^{-1} d z
$$

for any $\lambda$ sufficiently small, and it was shown in the proof of Lemma 3.4 that $P_{\lambda, \varepsilon} \rightarrow P_{0, \varepsilon}$ in the norm of operators on $\mathscr{H}_{n}$. Recall that

$$
A_{\lambda, \varepsilon}=\frac{\left(B_{\lambda, \varepsilon} T\right)^{2}-I}{\lambda^{2}}
$$

where $B_{\lambda, \varepsilon}$ is multiplication by $h\left((\lambda / 2) r^{2}\right) e^{(i / 2) \varepsilon \lambda^{2} r^{2}}$. Since $h$ is infinitely differentiable with bounded derivatives, which are $O\left(\left(1+t^{2}\right)^{-\alpha / 2}\right)$ as $t \rightarrow \infty$ for some $\alpha>0$ [7], and multiplication by $r^{2}$ is a bounded operator from $\mathscr{H}_{n}$ to $\mathscr{H}_{n-2}$ for $n \geqq 2$ [7] it follows from (3.17) and Lemma 3.4 that the map $\lambda \rightarrow P_{\lambda, \varepsilon} \in \mathscr{L}\left(\mathscr{H}_{n+2(k+1)}, \mathscr{H}_{n}\right)$ is $k$-times differentiable at $\lambda=0$ for $n=1,2, \ldots$ and all $k=0,1, \ldots$. Since we have

$$
\xi_{\lambda, \varepsilon}=P_{\lambda, \varepsilon} \xi_{0, \varepsilon}
$$

for $\lambda$ sufficiently small, and $\xi_{0, \varepsilon} \in \mathscr{H}_{n}$ for all $n=1,2, \ldots$, it follows that the map $\lambda \rightarrow \xi_{\lambda, \varepsilon} \in \mathscr{H}_{n}$ is infinitely differentiable at $\lambda=0$ for each $n=1,2, \ldots$.

Since as above $\lambda \rightarrow B_{\lambda, \varepsilon} \in \mathscr{L}\left(\mathscr{H}_{n+2(k+1)}, \mathscr{H}_{n}\right)$ is $k$-times differentiable at $\lambda=0$ for $n=1,2, \ldots$, and all $k=0,1, \ldots$, and $\lambda \rightarrow \xi_{\lambda, \varepsilon} \in \mathscr{H}_{n}$ is infinitely differentiable at $\lambda=0$ for all $n=1, \ldots$, it follows that we can differentiate both sides of Eq. (2.4) at $\lambda=0$, now written as

$$
A_{\lambda, \varepsilon} \xi_{\lambda, \varepsilon}=0,
$$

obtaining Eq. (2.9) rewritten in the space $\mathscr{H}_{n}$.

This finishes the proof of Theorem 2.1 when $E_{0}=0$.

\section{The Other Special Energies}

We now want to show how to extend the proof of Theorem 2.1, given in the previous section for the case $E_{0}=0$, to the case $E_{0}=2 \cos \pi p / q$. In this case (2.1) is rewritten as

$$
B_{\lambda, \varepsilon} T_{E_{0}} \xi_{\lambda, E_{0}, \varepsilon}=\xi_{\lambda, E_{0}, \varepsilon}
$$

where $\xi_{\lambda, E_{0}, \varepsilon}=\xi_{\lambda, E_{0}+\varepsilon \lambda^{2}}$ and $T_{E_{0}}=\mathscr{F} S_{E_{0}} \mathscr{F}^{-1}=B_{0}\left(E_{0}\right) T$.

If we try to repeat the proof of Sect. 3 we will notice that we cannot prove the crucial Lemma 3.2 because (3.4) is no longer true for $B_{\lambda, \varepsilon} T_{E_{0}}$, since $T_{E_{0}}$ is not unitary in $\mathscr{H}_{n}$. To avoid this problem we must change the norm of the spaces $\mathscr{H}_{n}$ to make $T_{E_{0}}$ unitary. This can be motivated as follows: recall that $S_{E_{0}}$ is unitary on $\mathscr{K}_{E_{0}}=L^{2}\left(\mathbf{R},\left(x^{2}-E_{0} x+1\right) d x\right)$, orthogonal on $\mathscr{K}_{E_{0}}^{\prime}$, for $-2<E_{0}<2$. Since $\mathscr{F} \times \mathscr{F}^{-1}=(2 / i) \partial$, where $(\partial f)\left(r^{2}\right)=f^{\prime}\left(r^{2}\right)$, we are led to define equivalent norms on $\mathscr{H}_{1}^{\prime}$ by

$$
\|f\|_{\mathscr{H}_{1, E_{0}}^{\prime}}^{2}=\operatorname{Re}\left\langle f\left(r^{2}\right),\left[\left(1-(2 / i) E_{0} \partial-4 \partial^{2}\right) f\right]\left(r^{2}\right)\right\rangle,
$$

the inner product being in $L^{2}([0, \infty), r d r)$. It follows that $\mathscr{F}$ is an orthogonal map 
from $\mathscr{K}_{E_{0}}^{\prime}$ to $\mathscr{H}_{1, E_{0}}^{\prime}$, and that $T_{E_{0}}$ is orthogonal on $\mathscr{H}_{1, E_{0}}^{\prime}$. Notice that $\mathscr{H}_{1,0}^{\prime}=\mathscr{H}_{1}^{\prime}$.

More generally, we let $\mathscr{H}_{0, E_{0}}=\mathscr{H}_{0}$, and for each $n=1,2, \ldots$ we define equivalent norms on the real Hilbert space $\mathscr{H}_{n}^{\prime}$, with the "primed" Hilbert spaces being defined as before, by

$$
\|f\|_{\mathscr{*}_{n, E_{0}}}^{2}=\operatorname{Re} \sum_{m=1}^{n}\left\langle f\left(r^{2}\right),\left[\left(1-(2 / i) E_{0} \partial-4 \partial^{2}\right)^{m} f\right]\left(r^{2}\right)\right\rangle_{m},
$$

$\langle,\rangle_{m}$ being the inner product in $L^{2}\left([0, \infty), r^{2 m-1} d r\right)$. We have $T_{E_{0}}$ orthogonal on $\mathscr{H}_{n, E_{0}}^{\prime}$.

This can be shown as follows. We can rewrite (4.1) as

$$
\|f\|_{\mathscr{K}_{1, E_{0}}^{\prime}}^{2}=\frac{1}{2 \pi} \operatorname{Re}\left\langle f\left(\varphi^{2}\right),\left(1-(2 / i) E_{0} \partial-4 \partial^{2}\right) f\left(\varphi^{2}\right)\right\rangle_{L^{2}\left(R^{2}, d^{2} \varphi\right)},
$$

and recall (see [7])

$$
\left(T_{E_{0}} f\right)\left(\varphi^{2}\right)=-e^{(i / 2) E_{0} \varphi^{2}} \int e^{i \varphi \cdot \varphi^{\prime}} \partial f\left(\varphi^{\prime 2}\right) \frac{d^{2} \varphi^{\prime}}{\pi},
$$

where $\varphi^{2}=\varphi \cdot \varphi, \partial=\partial / \partial \varphi^{2}$. We also write $P_{E_{0}}(x)=1-E_{0} x+x^{2}$. If $T_{E_{0}}^{t}$ denotes the transpose of $T_{E_{0}}$ with respect to the real part of the inner product on $L^{2}\left(\mathbf{R}^{2}, d^{2} \varphi\right)$, the orthogonality of $T_{E_{0}}$ on $\mathscr{H}_{1, E_{0}}^{\prime}$ is equivalent to

$$
T_{E_{0}}^{t} P_{E_{0}}\left(\frac{2}{i} \partial\right) T_{E_{0}}=P_{E_{0}}\left(\frac{2}{i} \partial\right)
$$

Notice that for $-2<E_{0}<2$ we can find strictly positive finite constants $C_{1}, C_{2}$ such that

$$
C_{1} P_{0}(x) \leqq P_{E_{0}}(x) \leqq C_{2} P_{0}(x)
$$

for all $x \in \mathbf{R}$. Thus

$$
C_{1} P_{0}\left(\frac{2}{i} \partial\right) \leqq P_{E_{0}}\left(\frac{2}{i} \partial\right) \leqq C_{2} P_{0}\left(\frac{2}{i} \partial\right)
$$

as operators on $L^{2}\left(\mathbf{R}^{2}, d^{2} \varphi\right)$. This expresses the equivalence of the norms in $\mathscr{H}_{1, E_{0}}^{\prime}$ and $\mathscr{H}_{1,0}^{\prime}$.

Similarly, we rewrite (4.2) as

$$
\|f\|_{\mathscr{F}_{n, E_{0}}^{\prime}}^{2}=\sum_{m=1}^{n} s_{m} \operatorname{Re}\left\langle f\left(\varphi^{2}\right),\left[\prod_{j=1}^{m} P_{E_{0}}\left(\frac{2}{i} \partial_{j}\right) f\right] \varphi^{2}\right\rangle_{L^{2}\left(\mathbf{R}^{2 m}, d^{2 m} \varphi\right)}
$$

where

$$
\begin{aligned}
\varphi & =\left(\varphi_{1}, \ldots, \varphi_{m}\right) \in \mathbf{R}^{2 m}, \varphi_{1}, \ldots, \varphi_{m} \in \mathbf{R}^{2}, \\
\varphi^{2} & =\varphi \cdot \varphi=\varphi_{1}^{2}+\cdots+\varphi_{m}^{2}, \partial_{j}=\frac{\partial}{\partial \varphi_{j}^{2}},
\end{aligned}
$$

and $s_{m}^{-1}=$ area of unit sphere in $\mathbf{R}^{2 m}$. 
For each $m=1, \ldots, n$ we can write (with the obvious identifications)

$$
T_{E_{0}}=\prod_{j=1}^{m} T_{E_{0}, j}
$$

each $T_{E_{0}, j}$ being given by (4.3) with respect to the variable $\varphi_{j}$ (see [11]). It follows from (4.4) and (4.6) that

$$
T_{E_{0}}^{t} \prod_{j=1}^{m} P_{E_{0}}\left(\frac{2}{i} \partial_{j}\right) T_{E_{0}}=\prod_{j=1}^{m} P_{E_{0}}\left(\frac{2}{i} \partial_{j}\right)
$$

with respect to the real part of the inner product in $L^{2}\left(\mathbf{R}^{2 m}, d^{2 m} \varphi\right)$, so $T_{E_{0}}$ is orthogonal on $\mathscr{H}_{n, E_{0}}^{\prime}$.

Similarly, it follows from (4.5) that the norms in $\mathscr{H}_{n, E_{0}}^{\prime}$ and $\mathscr{H}_{n, 0}^{\prime}$ are equivalent for $-2<E_{0}<2$. One can check that the norms of $\mathscr{H}_{n, 0}^{\prime}$ and $\mathscr{H}_{n}^{\prime}$ are also equivalent.

We now complexify $\mathscr{H}_{n, E_{0}}^{\prime}$ into $\mathscr{H}_{n, E_{0}}$. It follows that $T_{E_{0}}$ is unitary on each $\mathscr{H}_{n, E_{0}}$; and similarly, with $R_{E_{0}}=B_{0}\left(E_{0}\right) R$, we have $R_{E_{0}}, \mathscr{H}_{n, E_{0}}^{0}$, with the same properties as before.

The results of Sect. 3 are still valid, with obvious changes, if we replace $T$ by $T_{E_{0}}$ and $\mathscr{H}_{n}$ by $\mathscr{H}_{n, E_{0}}$, in case $E_{0}=2 \cos \pi p / q$, with $0<p<q$ relatively prime integers.

\section{Appendix A}

Let $X, Y$ be Banach spaces and let $0<t<1$. For $k \geqq 1$ we define $[X, Y]_{t}^{k}$ by

$$
\begin{aligned}
& {[X, Y]_{t}^{1}=[X, Y]_{t},} \\
& {[X, Y]_{t}^{k}=\left[[X, Y]_{t}^{k-1}, Y\right]_{t},}
\end{aligned}
$$

where $[X, Y]_{t}$ is the interpolation space between $X$ and $Y$ at level $t$.

If $X \subset Y$ as sets, $X \subset Y$ means that the injection from $X$ into $Y$ is continuous.

Lemma 5.1. There exists a constant $c$ such that for every $n \geqq 2$ and every $t>0$

$$
\left[\mathscr{H}_{0}, \mathscr{H}_{n}^{0}\right]^{[n c / t]} \subset \mathscr{H}_{n-1}^{0} .
$$

Proof. We first prove the result for $n=2$ and then proceed by induction. Let $Z_{s}$ be the Hilbert space

$$
Z_{s}=\left\{f:[0, \infty] \rightarrow \mathbf{C} ;\left\|r^{s-1 / 2} f\left(r^{2}\right)\right\|_{2}<\infty\right\} .
$$

We have $\mathscr{H}_{0}=Z_{0}$, and $\mathscr{H}_{2}^{0} \subseteq Z_{2}$. The interpolation between the spaces $Z_{s}$ is explicit (e.g., [9]); so we get that

$$
\left[\mathscr{H}_{0}, \mathscr{H}_{2}^{0}\right]_{t}^{k} \subset\left[Z_{0}, Z_{2}\right]_{t}^{k}=Z_{2\left(1-(1-t)^{k}\right)} .
$$

It follows that, if $k>\log 2 / t$ so $(1-t)^{k}<1 / 2$, we have $\left[\mathscr{H}_{0}, \mathscr{H}_{2}^{0}\right]_{t}^{k} \subseteq Z_{1}$.

Similarly, $R \mathscr{H}_{0}=\mathscr{H}_{0}=Z_{0}$ and $R \mathscr{H}_{2}^{0}=\mathscr{H}_{2}^{0} \subseteq Z_{2}$. So by the same argument $R\left[\mathscr{H}_{0}, \mathscr{H}_{2}^{0}\right]_{t}^{k} \hookrightarrow Z_{1}$ if $k>\log 2 / t$. But then $\left[\mathscr{H}_{0}, \mathscr{H}_{2}^{0}\right]_{t}^{k} \hookrightarrow \mathscr{H}_{1}^{0}$ since $\mathscr{H}_{1}^{0}=$ $\left\{f ; f \in Z_{1}\right.$ and $\left.R f \in Z_{1}\right\}$. This proves (5.1) for $n=1$.

Now let us fix $\bar{n}$ and assume (5.1) to be true for all $n \leqq \bar{n}$ with the same constant 
c. Then we know that

$$
\left[\mathscr{H}_{0}, \mathscr{H}_{\bar{n}+1}^{0}\right]_{t}^{k} \hookrightarrow\left[\mathscr{H}_{\bar{n}-1}^{0}, \mathscr{H}_{\bar{n}+1}^{0}\right]_{t}^{k-[c n / t]}
$$

for $k \geqq[c n / t]$. It thus suffices to consider the interpolation spaces $\left[\mathscr{H}_{\bar{n}-1}^{0}\right.$, $\left.\mathscr{H}_{\bar{n}+1}^{0}\right]_{t}^{k}$. Let, as before, $(\partial f)\left(r^{2}\right)=f^{\prime}\left(r^{2}\right)$. We then have that $\partial^{j}=\mathscr{H}_{\bar{n}-1} \rightarrow Z_{m}$ is a bounded operator for $m=1, \ldots, \bar{n}-1$ and $j=0, \ldots, m$, and also that $\partial^{j}: \mathscr{H}_{\bar{n}+1} \rightarrow Z_{n}$ is bounded for $m=1, \ldots, \bar{n}+1$ and $j=0, \ldots, m$. It follows by interpolation that for $j=0, \ldots, \bar{n}-1, \partial^{j}$ is a bounded operator from $\left[\mathscr{H}_{\bar{n}-1}^{0}, \mathscr{H}_{\bar{n}+1}^{0}\right]_{t}^{k}$ to $\left[Z_{\bar{n}-1}, Z_{\bar{n}+1}\right]_{t}^{k}=$ $Z_{\bar{n}+1-2(1-t)^{k}}$. Thus if $(1-t)^{k}<1 / 2$ we have that $\partial^{j}\left[\mathscr{H}_{\bar{n}-1}^{0}, \mathscr{H}_{\bar{n}+1}^{0}\right]_{t}^{k}$ is contained in $Z_{\bar{n}}$ for $i=0, \ldots, \bar{n}-1$. Similarly we can proceed in the same way with the operator $R$ and obtain

$$
R\left[\mathscr{H}_{\bar{n}-1}^{0}, \mathscr{H}_{\bar{n}+1}^{0}\right]_{t}^{k} \hookrightarrow Z_{\bar{n}-1-2(1-t)^{k}}
$$

In particular, if $(1-t)^{k}<1 / 2$, we get that

$$
R\left[\mathscr{H}_{\bar{n}-1}^{0}, \mathscr{H}_{\bar{n}+1}^{0}\right] \hookrightarrow Z_{\bar{n}} \text {. }
$$

Notice that the spaces $Z_{s}$ are not included into each other, but we used the fact that the spaces $\left[\mathscr{H}_{\bar{n}-1}^{0}, \mathscr{H}_{\bar{n}+1}^{0}\right]_{t}^{k}$ form a decreasing sequence in $k$ with respect to inclusion.

We have (see Eq. (3.5) in [7]) that

$$
\left\|r^{\bar{n}-1 / 2}(R f)\left(r^{2}\right)\right\|_{2}=\left\|2^{\bar{n}} r^{\bar{n}-1 / 2} \partial^{\bar{n}} f\left(r^{2}\right)\right\|_{2}
$$

Thus we can conclude that, if $(1-t)^{k}<1 / 2$, then

$$
\left[\mathscr{H}_{\bar{n}-1}^{0}, \mathscr{H}_{\bar{n}+1}^{0}\right]_{t}^{k} \subset \mathscr{H}_{\bar{n}}^{0}
$$

This finishes the proof.

\section{Appendix B}

Theorem 6.1. The unit sphere of $\mathscr{H}_{n+2}$ is percompact in $\mathscr{H}_{n}$ for $n \geqq 1$.

Proof. For simplicity we will consider the case $n=1$. The general case can be treated in the same way; one only needs to consider a larger number of derivatives.

Let $f \in \mathscr{H}^{3}$ with $\|f\|_{\mathscr{H}_{3}} \leqq 1$. We will establish a series of bounds. First, let $r \geqq 1$. Then we have

$$
\begin{aligned}
\left|f\left(r^{2}\right)\right| & =\left|2 \int_{r^{2}}^{\infty} t f^{\prime}\left(t^{2}\right) d t\right| \\
& \leqq\left\|2 r^{5 / 2} f^{\prime}(r)\right\|_{2}\left(\int_{r^{2}}^{\infty} t^{-3} d t\right)^{1 / 2} \leqq \frac{1}{\sqrt{2} r^{2}} \\
\left|f^{\prime}\left(r^{2}\right)\right| & =\left|2 \int_{r^{2}}^{\infty} t f^{\prime \prime}\left(t^{2}\right) d t\right| \\
& \leqq \frac{1}{2}\left\|4 r^{5 / 2} f^{\prime \prime}\left(r^{2}\right)\right\|_{2}\left(\int_{r^{2}}^{\infty} t^{-3} d t\right)^{1 / 2} \leqq \frac{1}{2 \sqrt{2} r^{2}}
\end{aligned}
$$


For $0 \leqq r \leqq 1$ we have

$$
\begin{aligned}
\left|f\left(r^{2}\right)\right| & =\left|f(1)-2 \int_{r^{2}}^{1} t f^{\prime}(t) d t\right| \\
& \leqq \frac{1}{\sqrt{2}}+\left\|2 r^{1 / 2} f^{\prime}\left(r^{2}\right)\right\|_{2} \int_{r^{2}}^{1} t d t \leqq C_{1} \\
\left|f^{\prime}\left(r^{2}\right)\right| & =\left|f^{\prime}(1)-2 \int^{1} r^{2} t f^{\prime \prime}(t) d t\right| \\
& \leqq \frac{1}{2 \sqrt{2}}+\frac{1}{2}\left\|4 r^{3 / 2} f^{\prime \prime}\left(r^{2}\right)\right\|_{2}\left(\int_{r^{2}}^{1} t^{-1} d t\right)^{1 / 2} \\
& \leqq C_{2}(|\log r|)^{1 / 2}+C_{3} ;
\end{aligned}
$$

where $C_{1}, C_{2}, C_{3}$ are finite constants, independent of $f$.

Now let $0 \leqq R_{1} \leqq R_{2}$; we define

$$
\begin{aligned}
\|f\|_{1, R_{1}, R_{2}}^{2}= & \left\|r^{1 / 2} f\left(r^{2}\right) \chi\left(R_{1}<r<R_{2}\right)\right\|_{2}^{2} \\
& +\left\|2 r^{1 / 2} f^{\prime}\left(r^{2}\right) \chi\left(R_{1}<r<R_{2}\right)\right\|_{2}^{2} .
\end{aligned}
$$

For $m \geqq 1$ we let

$$
\|f\|_{\mathscr{H}_{1}}^{2}=\|f\|_{1,0,1 / m}^{2}+\|f\|_{1,1 / m, m}^{2}+\|f\|_{1, m, \infty}^{2} .
$$

Using (6.1),(6.2),(6.3) and (6.4) we get

$$
\|f\|_{1,0,1 / m}^{2} \leqq C_{4} \int_{0}^{1 / m} r d r+C_{5} \int_{0}^{1 / m} r|\log r| d r \leqq \frac{C_{6}}{m}
$$

and

$$
\|f\|_{1, m, \infty}^{2} \leqq C_{7} \int_{m}^{\infty} r^{-3} d r \leqq \frac{C_{8}}{m^{2}},
$$

where again the $C$ 's denote finite constants.

Let us now consider a sequence $f_{k}$ in the unit ball of $\mathscr{H}_{3}$. We will show that we can extract a subsequence converging in $\mathscr{H}_{1}$.

To do so we will first show that for any $m=1,2, \ldots$ we can find a subsequence $f_{k_{i}(m)}$ which is Cauchy with respect to the seminorm $\|\cdot\|_{1,1 / m, m}$, and we will construct $f_{k_{i}(m+1)}$ as a subsequence of $f_{k_{i}(m)}$.

To do this we apply the Arzela-Ascoli Theorem. Indeed, $f_{k}\left(r^{2}\right)$ and $f_{k}^{\prime}\left(r^{2}\right)$ are uniformly bounded and equicontinuous on $[1 / m, m]$. The uniform boundedness follows from (6.1)-(6.4). The equicontinuity follows from the bounds, valid for $f$ in the unit sphere of $\mathscr{H}_{3}$ :

$$
\begin{aligned}
\left|f\left(r_{2}^{2}\right)-f\left(r_{1}^{2}\right)\right| & =\left|2 \int_{r_{1}^{2}}^{r_{2}^{2}} t f^{\prime}(t) d t\right| \\
& \leqq\left(\int_{r_{1}^{2}}^{r_{2}^{2}} t d t\right)^{1 / 2}\left\|2 r^{1 / 2} f^{\prime}\left(r^{2}\right)\right\|_{2} \\
& \leqq\left(\frac{r_{2}^{4}-r_{1}^{4}}{2}\right)^{1 / 2},
\end{aligned}
$$




$$
\begin{aligned}
\left|f^{\prime}\left(r_{2}^{2}\right)-f^{\prime}\left(r_{1}^{2}\right)\right| & =\left|2 \int_{r_{1}^{2}}^{r_{2}^{2}} t f^{\prime \prime}\left(t^{2}\right) d t\right| \\
& \leqq \frac{1}{2}\left(\int_{r_{1}^{2}}^{r_{2}^{2}} t^{-1} d t\right)^{1 / 2}\left\|4 r^{3 / 2} f^{\prime \prime}\left(r^{2}\right)\right\|_{2} \\
& \leqq \frac{1}{2}\left(\log r_{2}^{2}-\log r_{1}^{2}\right)^{1 / 2}
\end{aligned}
$$

We can thus define the sequence $f_{k_{i}(m)}$ by induction on $m$. Indeed, by Arzela-Arcoli we can extract a subsequence $f_{k_{i}(m+1)}$ of $f_{k_{i}(m)}$ such that $f_{k_{i}(m+1)}\left(r^{2}\right)$ and $f_{k_{i}(m+1)}^{\prime}\left(r^{2}\right)$ are Cauchy in the sup norm in $[1 / m+1, m+1]$ and hence Cauchy with respect to the seminorm $\|\cdot\|_{1,1 /(m+1), m+1}$. We then apply the usual diagonalization procedure obtaining the subsequence $f_{k_{m}(m)}$ that converges with respect to the norm of $\mathscr{H}_{1}$ by (6.5)-(6.7).

\section{References}

1. Bougerol, P., Lacroix, J.: Products of random matrices with applications to Schrödinger operators. Boston: Birkhäuser 1985

2. Kappus, M., Wegner, Z.: Anomaly in the band centre of the one-dimensional Anderson model. Z. Phys. B45, 15-21 (1981)

3. Thouless, D.: In: Ill-Condensed Matter. Balian, R., Maynard, R., Toulouse, G. (eds.). Amsterdam: North Holland 1979

4. Derrida, B., Gardner, E.: Lyapunov exponent of the one dimensional Anderson model: Weak disorder expansions. J. Phys. 45, 1285-1295 (1984)

5. Bovier, A., Klein, A.: Weak disorder expansion of the invariant measure for the one-dimensional Anderson model. J. Stat. Phys. 51, 501-517 (1988)

6. Klein, A., Speis, A.: Regularity of the invariant measure and of the density of states in the one dimensional Anderson model. J. Funct. Anal. 88, 211-227 (1990)

7. Campanino, M., Klein, A.: A supersymmetric transfer matrix and differentiability of the density of states in the one-dimensional Anderson model. Commun. Math. Phys. 104, 227-241 (1986)

8. March, P., Sznitman, A.: Some connections between excursion theory and the discrete random Schrödinger equation with applications to analyticity and smoothness properties of the density of states in one dimension (preprint)

9. Reed, M., Simon, B.: Methods of modern mathematical physics, II. Fourier analysis, self-adjointness. New York: Academic Press 1975

10. Chernoff, P.: Product formulas, nonlinear semigroups, and addition of unbounded operators. Mem. Am. Math. Soc. 140; Providence, RI: Am. Math. Soc. 1974

11. Klein, A., Speis, A.: Smoothness of density of states in the Anderson model on a one-dimensional strip. Ann. Phys. 183, 352-398 (1988)

\section{Communicated by T. Spencer}

Received May 18, 1989 